\title{
Machine learning for detailed chemistry reduction in DNS of a syngas turbulent oxy-flame with side-wall effects
}

\author{
Kaidi Wan ${ }^{\mathrm{a}}$, Camille Barnaud ${ }^{\mathrm{a}}$, Luc Vervisch ${ }^{\mathrm{a}, *}$, Pascale Domingo $^{\mathrm{a}}$
}

${ }^{a}$ CORIA - CNRS, Normandie Université, INSA de Rouen, 76801 Saint-Etienne-du-Rouvray, France

\begin{abstract}
A novel chemistry reduction strategy based on convolutional neural networks (CNNs) is developed and applied to direct numerical simulation (DNS) of a turbulent non-premixed flame interacting with a cooled wall. The fuel syngas mixture is burning in pure oxygen. The training and the subsequent application of the CNN rely on the processing of two-dimensional (2D) images built from species mass fractions and temperature (CNN input), to predict the corresponding chemical sources at the center of the image (CNN output). This image-type treatment of chemistry is found to efficiently capture intermediate radicals species highly sensitive to the local flame topology. To reduce the CPU cost, a simplified 2D DNS database with detailed chemistry serves as reference and is used for training and testing the neural network. Comparisons are also made a posteriori against the same 2D DNS with a reduced chemical scheme specialized for syngas. Then, three-dimensional (3D) DNS are conducted either with CNN or the reduced chemistry for more a posteriori tests. The CNN reduced chemistry outperforms the reduced Arrhenius based mechanism in the prediction of radical species, such as monoatomic hydrogen, and also in terms of CPU cost.
\end{abstract}

Keywords: Convolutional neural network, Chemistry reduction, Direct numerical simulation, Syngas

${ }^{*}$ Corresponding author

Email address: vervisch@coria.fr (Luc Vervisch) 


\section{Introduction}

The chemical mechanisms for hydrocarbon fuel combustion can involve up to hundreds of species and thousands of reactions [1]. Because of the very large number of degrees of freedom to be solved, such fully detailed mechanisms can hardly be introduced in threedimensional (3D) numerical simulations of flames. In this context, the downsizing of chemical kinetics, still securing the prediction of given major thermochemical properties, is mandatory for virtual prototyping of real combustion systems, particularly those involving heavy hydrocarbon liquid or solid fuels [2].

Numerous successful strategies have been discussed in the literature to reduce detailed chemical kinetics. They are based mainly on fuel lumping, time-scale and principal component analysis or direct numerical treatment of the chemical responses from graph analysis or genetic algorithms, sometimes associated with tabulation methods [3-17].

Learning from a database to then reproduce the chemical response has received extensive attention in tabulation of combustion chemistry. Artificial neural networks (ANNs) were found accurate, and both CPU and memory efficient [18, 19]. Along similar lines, convolutional neural networks (CNNs), which were originally developed for analyzing visual representations [20], have been introduced in turbulent combustion modeling for direct deconvolution of the filtered progress variable [21], for modeling the unresolved flame surface wrinkling [22] and for the direct reconstruction of unresolved sources and fluxes from mesh-resolved quantities in large-eddy simulation (LES) [23]. Compared to ANNs, CNNs reduce the number of connections per layer to stack more of them efficiently, thus increasing the depth of the neural network.

In the present study, a novel chemistry reduction approach based on CNNs is proposed and also coupled with a three-dimensional direct numerical simulation (DNS) for validation. A turbulent non-premixed oxy-flame interacting with a cooled wall is considered. This configuration covers a wide range of combustion and turbulence conditions in terms of equivalence ratio, progress of reaction and enthalpy including thermal and viscous boundary layers effects, making it a relatively generic test case. The syngas fuel composition is representative of a $\mathrm{CO}_{2}$ free blast-furnace exhaust flux, as found in a recycling combustion process. The input of the CNN are two-dimensional (2D) images of the species mass fractions and temperature, while the output are the corresponding chemical burning rates at the center point of the image. The training is performed from a simplified two-dimensional planar DNS, with detailed chemistry and input images built from a subset of the full species vector. This subset of 11 species is chosen to match the major and minor species retained in a reduced chemical mechanism optimized for syngas. Once the CNN trained, this reduced set of species becomes the input to get the corresponding chemical rates. Then, only this reduced set of species needs to be transported with the flow, saving much CPU time and reducing memory requirement.

The objective is to test the feasibility of the novel method, which should be applicable to any reference detailed chemical mechanism. The generic GRI-3.0 detailed mechanism [24] without $\mathrm{NO}_{x}$ (217 elementary reactions and 35 chemical species) is used in this first attempt. Once trained for reproducing the chemical sources of a reduced set of 11 species, the CNN for chemistry reduction is tested a priori against the detailed chemistry DNS. Then, the obtained neural network is fully coupled with the flow solution for two- and threedimensional DNS of the non-premixed flame-wall interaction. The two-dimensional cases are with the detailed chemistry, the reduced chemical mechanism and the CNN chemistry. The three-dimensional cases are with the $\mathrm{CNN}$ and the reduced chemistry.

\section{Turbulent flame configuration and numerics}

A turbulent non-premixed oxy-flame flame under a pressure of $341.3 \mathrm{kPa}$ and featuring side-wall effects is simulated (Fig. 11). A representative syngas composition is injected through the upper inlet (width of $2 \mathrm{~mm}$ ) at $1223 \mathrm{~K}$ and bulk velocity of $50 \mathrm{~m} / \mathrm{s}$, with $\mathrm{H}_{2} / 0.0085$, $\mathrm{CO} / 0.7852, \mathrm{CO}_{2} / 0.0514, \mathrm{~N}_{2} / 0.1549$ in mass fraction. Below the splitter plate of thickness $0.2 \mathrm{~mm}$, the lower inlet (width of $10 \mathrm{~mm}$ ) supplies pure oxygen at $298 \mathrm{~K}$ and $100 \mathrm{~m} / \mathrm{s}$. A preliminary simulation of an oxygen turbulent periodical 3D channel flow at the oxygen bulk velocity serves as a database to impose a proper turbulent inlet to the oxygen stream. A laminar channel flow profile is imposed for the fuel stream, between the wall and the splitter plate. A non-slip wall is placed at the top of the domain while a symmetrical boundary condition is used at the bottom. Periodic boundary conditions are employed in the spanwise $z$ direction (Fig. 1) and a convective boundary condition is used for the outflow. The wall temperature $T_{w}(\underline{x}, t)$ is nonuniform and computed assuming that the heat flux locally reaching the wall is evacuated on its other side by an efficient water cooling with a convective heat transfer $h=3300 \mathrm{~W} \cdot \mathrm{m}^{-2} \cdot \mathrm{K}^{-1}$, with a water temperature $T_{o}=353 \mathrm{~K}$ (i.e., $h\left(T_{w}-T_{o}\right)=\lambda\left(\partial T / \partial n_{w}\right)_{g a s}, n_{w}$ is the 


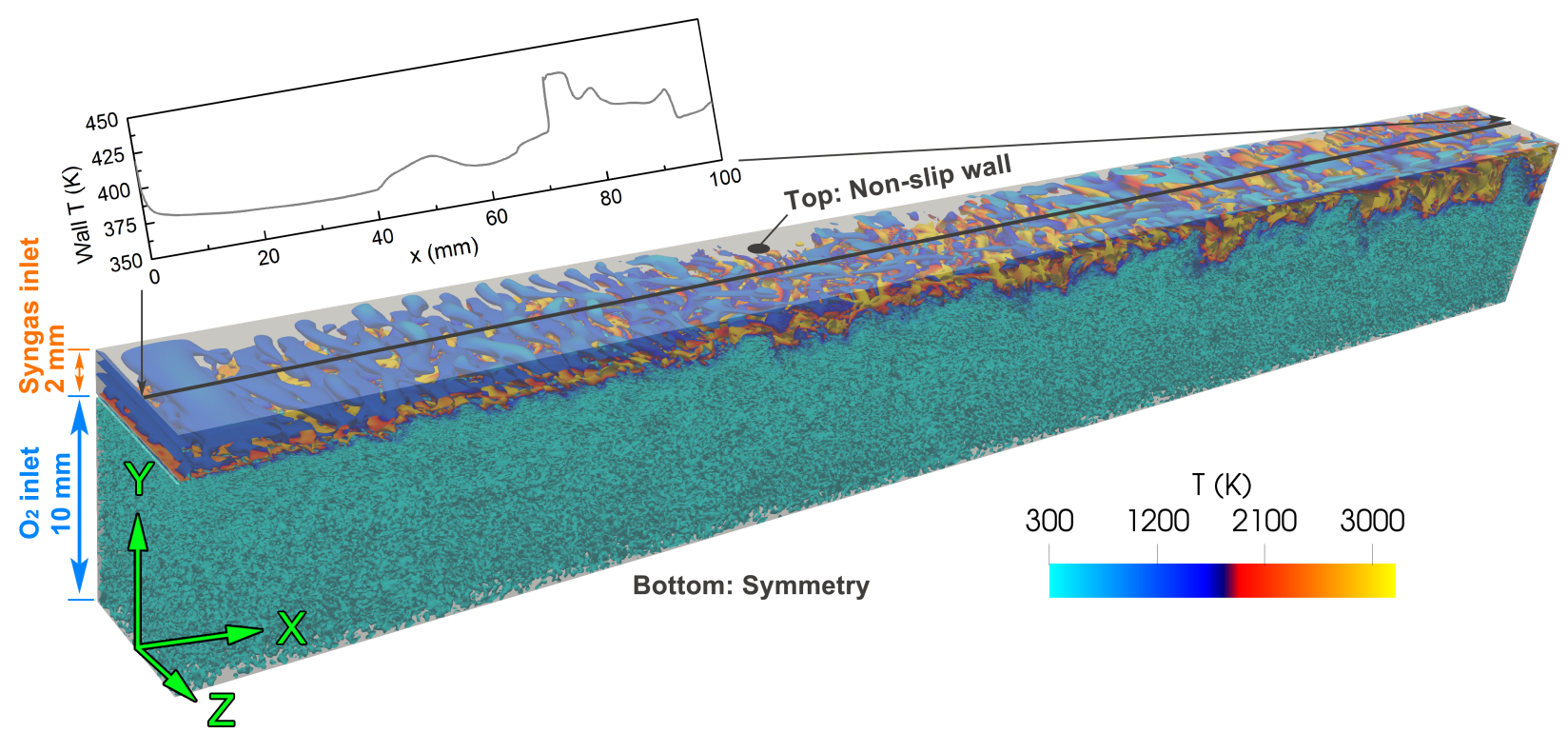

Figure 1: Instantaneous iso-surface of $Q$ criterion [25] predicted by CNN-DNS: $Q=10^{7} \mathrm{~s}^{-2}$, colored by gas temperature. Top graph: distribution of instantaneous wall temperature along the centreline.

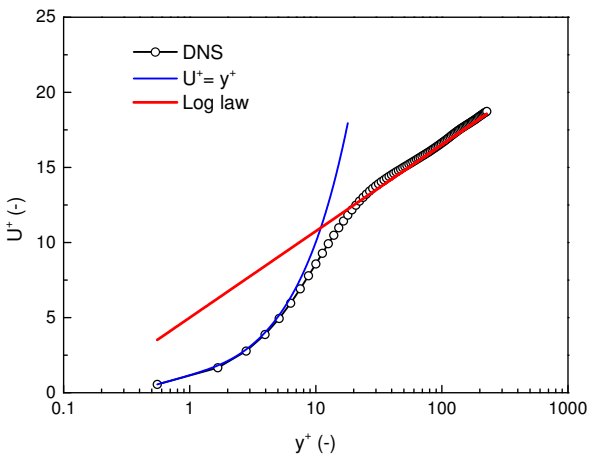

Figure 2: Velocity distribution in wall units for 3D non-reactive case (mesh verification).

wall normal direction). The physical dimensions of the computational domain composed of $66.44 \mathrm{M}$ cells are $L_{x}=100 \mathrm{~mm}, L_{y}=12 \mathrm{~mm}$ and $L_{z}=7.68 \mathrm{~mm}$. The mesh is uniform in $x$ (streamwise) and $z$ (spanwise, not considered in 2D) directions, with the grid spacing $h_{x}$ $=h_{z}=60 \mu \mathrm{m}$. In the $y$ (vertical) direction, the mesh keeps a fine resolution of $20 \mu \mathrm{m}$ for the upper region close to the wall $(y>10 \mathrm{~mm})$ where the flame is located ${ }^{1}$ The mesh gets coarser as $y$ decreases from 10 to $0 \mathrm{~mm}$ to reach a grid spacing of $100 \mu \mathrm{m}$ at the bottom of the computational domain. The first point off the

\footnotetext{
${ }^{1}$ Simulations of one-dimensional canonical strained non-premixed flames were conducted to determine the grid resolution for the present free stream conditions.
}

top wall is at $y^{+}=0.55$ where the superscript + denotes the usual non-dimensionalization by the viscous length scales. There are 9 grid points within $y^{+}=10$ to resolve the viscous layer near the wall [26]. It was verified from a non-reactive simulation that the structure of the wall boundary layer agrees with what is expected (Fig. 2). The time step is fixed at $0.3 \mu$ s $(\mathrm{CFL} \approx 0.8)$ with subiteration in a stiff-chemistry ODE solver.

The governing equations for the gas mixture are solved with a low-Mach number in-house code [2731]. The conservation equations for mass, momentum, species and temperature of the gas phase are those of [31], here without the source terms due to coal particles. The flow viscosity and the thermal conductivity of the gas mixtures are computed from Wilke's type mixing rules. The Lewis number is assumed to be unity for all the species.

The numerics is based on an approach previously used for both DNS and LES [32, 33]. The time advancement relies on a second-order Crank-Nicolson scheme. A bounded quadratic upstream interpolation for convective kinematics (BQUICK) scheme is used for the scalar advection terms in the species and temperature equations, while a second-order central difference scheme is applied to the scalar diffusion terms in the species and temperature equations and all terms in the momentum equation. An alternating direction implicit (ADI) method has been employed, in which semiimplicit tridiagonal/pentadiagonal equations are solved 
Table 1: Structure of the sequential CNNs used (see [34 for details on functions).

\begin{tabular}{lll}
\hline Layer type & $\begin{array}{l}\text { Activation } \\
\text { function }\end{array}$ & Output Shape \\
\hline Input & - & (None, 3, 3, 11) \\
Conv2D & ReLU & (None, 3, 3, 64) \\
MaxPooling2D & - & (None, 2, 2, 64) \\
Conv 2D & ReLU & (None, 2, 2, 256) \\
MaxPooling2D & - & (None, 1, 1, 256) \\
Flatten & - & (None, 256) \\
Dense & ReLU & (None, 256) \\
Dense & ReLU & (None, 64) \\
Dense & ReLU & (None, 16) \\
Dense (Output) & - & (None, 11) \\
\hline
\end{tabular}

separately for each direction.

\section{CNN chemistry reduction methodology}

\subsection{Training phase}

Figure 3 sketches the overall $\mathrm{CNN}$ training process from the $2 \mathrm{D}$ database, which is applied to the species $\mathrm{O}, \mathrm{O}_{2}, \mathrm{H}, \mathrm{H}_{2}, \mathrm{OH}, \mathrm{HO}_{2}, \mathrm{H}_{2} \mathrm{O}, \mathrm{H}_{2} \mathrm{O}_{2}, \mathrm{CO}$ and $\mathrm{CO}_{2}$ $\left(\mathrm{NO}_{x}\right.$ being not included, no source is computed for $\mathrm{N}_{2}$ ). Because the syngas is preheated to $1223 \mathrm{~K}$, rapid ignition occurs after mixing with pure oxygen and a nonpremixed flame develops in the computational domain (Fig. 1). After the flame is established, the simulation proceeds for 2 flow-through times $(2 \mathrm{~ms})$ based on the bulk velocity of the $\mathrm{O}_{2}$ stream.

The training database is built saving every $50 \mu$ s the 0.52 million 2D DNS fields of species mass fractions and temperature together with their sources, leading to 40 snapshots each containing 520,000 data. To augment the database up to 80 snapshots, the input data are perturbed applying $2 \%$ of uncorrelated random noise. Then, over the 80 snapshots, one of every 5 is selected to form the subsequent testing database (16 snapshots), while the left 64 snapshots constitute the training database.

In order to train the $\mathrm{CNN}$ with the two-dimensional simulations, a 2D test-box is constructed around every $i$-th DNS node with its neighboring 8 nodes (Fig. 3 ). Selecting the 8 neighboring nodes with the target node at the center, to form a $3 \times 32 \mathrm{D}$ image, appears as the most efficient procedure with the smallest size of CNN input. This test-box is used to built the $i$-th image composed of 9 points from the 2D distributions of the vector $\phi\left(x_{\ell}, t\right)=\left(Y_{1}\left(x_{\ell}, t\right), \cdots, Y_{N}\left(x_{\ell}, t\right), T\left(x_{\ell}, t\right)\right)$ for $\ell=1, \cdots, 9$, which contains information on the $N=10$ species mass fractions and temperature $\left(\mathrm{N}_{2}\right.$ not included). The "target" (or "label") of each image is the vector of the reaction rates at the $x$-centred $i$-th point, $\underline{\dot{\omega}}[i]=\left(\phi(\underline{x}, t+\delta t)-\phi^{\star}(\underline{x}, t)\right) / \delta t$, with $\delta t=0.3 \mu \mathrm{s}$

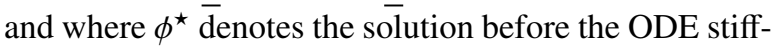
chemistry solver is applied. Casting the "targets" (i.e., the sources) in this form presents many advantages. Indeed, the output of the CNN can be used either as a chemical source $\underline{\dot{\omega}}[i]$ for integration, or directly to read from the $\mathrm{CNN} \phi(t+\delta t)=\underline{\dot{\omega}}[i] \times 0.3 \cdot 10^{-6}+\phi^{\star}(t)$ in a flow solver relying on splitting for chemistry integration where $\phi^{\star}(t)$ is known. In the latter option, sub-iterations may be needed for $\delta t>0.3 \mu$ s. In our simulations, the time step stays the same between training and usage of the CNN, therefore no linear approximation is actually performed. However, care should be taken that splitting method for chemistry integration can decrease the overall order of accuracy of the solver.

To secure the atomic mass balance over a reduced set of species whose sources $\dot{\omega}_{i}$ are learned from a detailed mechanism, the following procedure is adopted. (In practice, over the computational domain, the total default mass to be corrected represents only $0.016 \%$ of the total mass.) First, the atomic sources mass default are computed for carbon, hydrogen and oxygen. Let us denote $\alpha_{A, i}$ the number of atom $\mathrm{A}$ (i.e., $\mathrm{A} \equiv \mathrm{C}, \mathrm{H}$ or $\mathrm{O})$ in the $i$-th species. The mass imbalance in A source may be written $\Delta \dot{\omega}_{\mathrm{A}}=\sum_{i=1}^{N}\left[\left(\alpha_{\mathrm{A}, i} W_{\mathrm{A}}\right) / W_{i}\right] \dot{\omega}_{i}$, where $W_{\mathrm{A}}$ and $W_{i}$ are molar weights. Second, sources of carbon containing species of the syngas $\left(\mathrm{CO}\right.$ and $\left.\mathrm{CO}_{2}\right)$ and hydrogen containing species $\left(\mathrm{H}_{2}, \mathrm{H}, \mathrm{H}_{2} \mathrm{O}, \mathrm{OH}, \mathrm{HO}_{2}\right.$ and $\left.\mathrm{H}_{2} \mathrm{O}_{2}\right)$ are corrected adding $\Delta \dot{w}_{i}=-\left(Y_{i} / Y_{\mathrm{A}}\right) \Delta \dot{\omega}_{\mathrm{A}}$, with the atom mass fraction $Y_{\mathrm{A}}=\sum_{i=1}^{N} \alpha_{\mathrm{A}, i}\left(W_{A} / W_{i}\right) Y_{i}$. For oxygen atom, the correction is applied only to $\mathrm{O}_{2}$ and with the already corrected sources of $\mathrm{C}$ and $\mathrm{H}$ containing species. Then, atomic budgets are perfectly preserved by the chemical sources learned by the CNN.

$46 \%$ of the images have a central node temperature below $320 \mathrm{~K}$, these points with negligible sources are dropped out from the database. This leads to a final training database of $14.6 \mathrm{~GB}$ and a testing database of 3.7 GB. All data contributing to the "images" and to the "targets" are normalized by their respective maximum absolute value over all the database, these 22 maximum levels are stored (10 species mass fractions, temperature and sources $-\mathrm{N}_{2}$ not involved). The complete structure of the sequential CNNs is summarized in Table 1. The CNN architecture is inspired from [23], with two additional dense layers to secure the performance of the regression task required here. In total, the neural network 


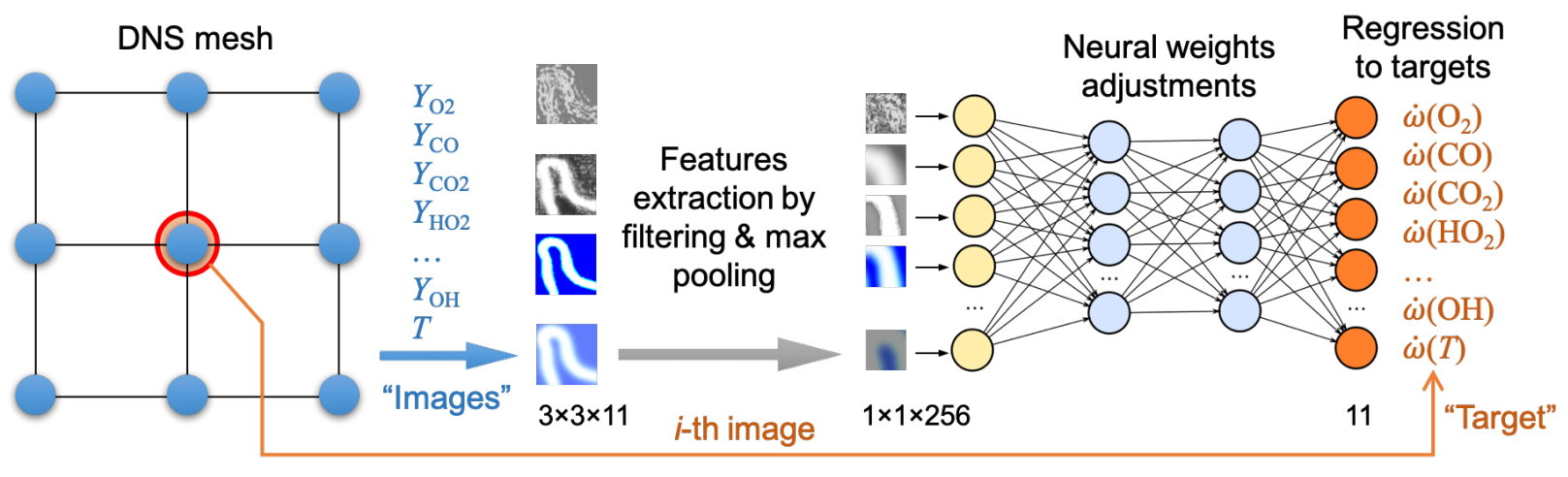

Figure 3: CNN training from 2D database.

contains 237,579 weights, which need to be adjusted.

To improve the precision of the CNNs for small values of the chemical sources, a second network with exactly the same structure is trained for a subset of the database defined by $Y_{\mathrm{O}_{2}}>0.9$ at the central node (i.e., where overall the burning rates stay very low). The subset-database is normalized based on its maximum absolute values. In the following, the networks are referred to as the main $\mathrm{CNN}$ and the second CNN (subset for lower burning rates).

The training of the CNNs is performed using the TensorFlow Python library with GPU support (www.tensorflow.org). A series of convolution/sampling operations are performed iteratively, in which the neural weights are adjusted until a satisfying minimal error is achieved between the "target" sources and the ones predicted by the CNNs. The Adam optimizer [35] is employed with a meansquared-error (MSE) loss function comparing the predictions to the target. A training step is performed with a batch of 10240 "images", leading to 1740 steps per "epoch"/iteration for the main CNN. The learning rate [34] is initially set to 0.001 to decay versus the steps $(n)$ according to $l_{r}=0.001 /(1.0+0.0001 \cdot \mathrm{n})$. The network converges in approximatively 500 epochs, requiring for the main CNN 15 hours on an Nvidia GeForce GTX 1080 Ti GPU.

\subsection{Testing phase}

The performance of the machine learning reduced chemistry is first examined in a-priori manner by comparing chemical sources from CNN and GRI-3.0. The part of the database unseen during training is probed. The mean-square error between the predictions and the targets is $1.5 \times 10^{-7}$ for the main $\mathrm{CNN}$ and $4.5 \times 10^{-8}$ for the second CNN. Figure 4 displays the predicted

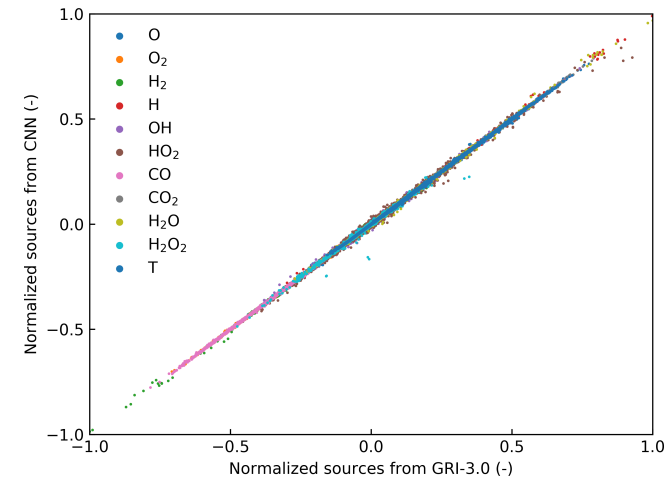

Figure 4: Comparison of the main CNN prediction on normalized sources of the 10 species and temperature against the GRI-3.0 target values for the testing database unseen during training.

chemical sources against their reference values for the main $\mathrm{CNN}$, confirming the accuracy of the approach. The second CNN achieves similar performance in this a-priori validation test (not shown for brevity).

\section{CNN-DNS full coupling}

The CNN reduced chemistry is now employed in the simulation of the syngas non-premixed flame (only CPU is used in this part with CNNs exported in C language, see supplementary material). Simulations are then performed with detailed chemistry (2D), CNN chemistry (2D and 3D) and a reduced chemistry (2D and 3D) given in Table 2. This reduced mechanism was derived from GRI-3.0 for the present syngas operating condition including wall heat loss effects, with the automated approach reported in detail in [13, 36] $!^{2}$

\footnotetext{
${ }^{2}$ This method combines a stochastic probing of the detailed chemistry response with directed relation graphs analysis with error prop-
} 


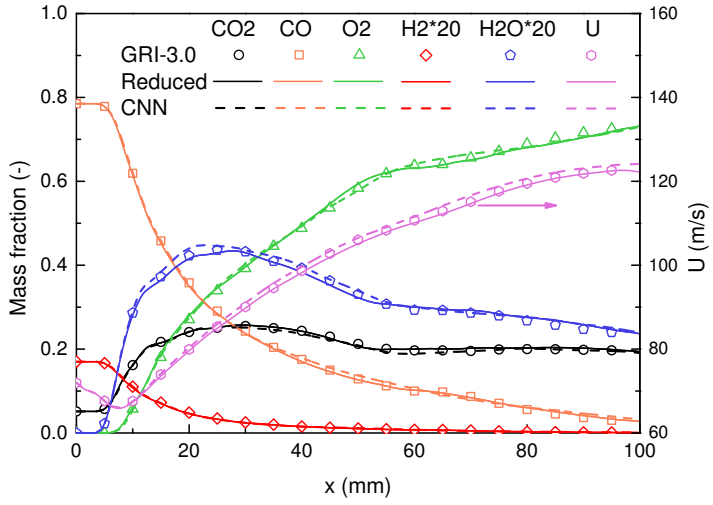

Figure 5: Averaged distributions of streamwise velocity and major species mass fractions: $\mathrm{CO}_{2}, \mathrm{CO}, \mathrm{O}_{2}, \mathrm{H}_{2}$ and $\mathrm{H}_{2} \mathrm{O}$ along the planar fuel-jet centreline $(y=11 \mathrm{~mm})$. Symbols: GRI-3.0. Solid line: Reduced mechanism (Table 2, Dashed line: CNN chemistry. 2D case.

Table 2: Reduced chemistry for syngas oxy-flame. Units are mol, s, $\mathrm{cm}^{3}$, cal and K. The Chaperon efficiencies of the GRI-3.0 mechanism are preserved for both three-body and fall-off reactions (see also supplementary material)

\begin{tabular}{|c|c|c|c|c|}
\hline $\mathrm{n}^{o}$ & Reaction & $A$ & $\beta$ & $E_{a}$ \\
\hline 1 & $2 \mathrm{O}+\mathrm{M} \rightleftharpoons \mathrm{O}_{2}+\mathrm{M}$ & $1.20 \mathrm{e}+17$ & -1.00 & 0 \\
\hline 2 & $\mathrm{O}+\mathrm{H}_{2} \rightleftharpoons \mathrm{H}+\mathrm{OH}$ & $3.87 \mathrm{e}+04$ & 2.70 & 6260 \\
\hline 3 & $\mathrm{O}+\mathrm{HO}_{2} \rightleftharpoons \mathrm{OH}+\mathrm{O}_{2}$ & $2.00 \mathrm{e}+13$ & 0 & 0 \\
\hline 4 & $\mathrm{O}+\mathrm{CO}(+\mathrm{M}) \rightleftharpoons \mathrm{CO}_{2}(+\mathrm{M})$ & $1.80 \mathrm{e}+10$ & 0 & 2385 \\
\hline 5 & $\mathrm{O}_{2}+\mathrm{CO} \rightleftharpoons \mathrm{O}+\mathrm{CO}_{2}$ & $2.50 \mathrm{e}+12$ & 0 & 47800 \\
\hline 6 & $\mathrm{H}+\mathrm{O}_{2}+\mathrm{M} \rightleftharpoons \mathrm{HO}_{2}+\mathrm{M}$ & $2.80 \mathrm{e}+18$ & -0.86 & 0 \\
\hline 7 & $\mathrm{H}+\mathrm{O}_{2}+\mathrm{N}_{2} \rightleftharpoons \mathrm{HO}_{2}+\mathrm{N}_{2}$ & $2.60 \mathrm{e}+19$ & -1.24 & 0 \\
\hline 8 & $\mathrm{H}+2 \mathrm{O}_{2} \rightleftharpoons \mathrm{HO}_{2}+\mathrm{O}_{2}$ & $2.08 \mathrm{e}+19$ & -1.24 & 0 \\
\hline 9 & $\mathrm{H}+\mathrm{O}_{2}+\mathrm{H}_{2} \mathrm{O} \rightleftharpoons \mathrm{HO}_{2}+\mathrm{H}_{2} \mathrm{O}$ & $1.13 \mathrm{e}+19$ & -0.76 & 0 \\
\hline 10 & $\mathrm{H}+\mathrm{O}_{2} \rightleftharpoons \mathrm{O}+\mathrm{OH}$ & $2.65 \mathrm{e}+16$ & -0.67 & 17041 \\
\hline 11 & $\mathrm{H}+\mathrm{OH}+\mathrm{M} \rightleftharpoons \mathrm{H}_{2} \mathrm{O}+\mathrm{M}$ & $2.20 \mathrm{e}+22$ & -2.00 & 0 \\
\hline 12 & $\mathrm{H}+\mathrm{HO}_{2} \rightleftharpoons \mathrm{O}_{2}+\mathrm{H}_{2}$ & $4.48 \mathrm{e}+13$ & 0 & 1068 \\
\hline 13 & $\mathrm{H}+\mathrm{HO}_{2} \rightleftharpoons 2 \mathrm{OH}$ & $8.40 \mathrm{e}+13$ & 0 & 635 \\
\hline 14 & $\mathrm{OH}+\mathrm{H}_{2} \rightleftharpoons \mathrm{H}+\mathrm{H}_{2} \mathrm{O}$ & $2.16 \mathrm{e}+08$ & 1.51 & 3430 \\
\hline 15 & $2 \mathrm{OH}(+\mathrm{M}) \rightleftharpoons \mathrm{H}_{2} \mathrm{O}_{2}(+\mathrm{M})$ & $7.40 \mathrm{e}+13$ & -0.37 & 0 \\
\hline 16 & $\mathrm{OH}+\mathrm{HO}_{2} \rightleftharpoons \mathrm{O}_{2}+\mathrm{H}_{2} \mathrm{O}$ & $1.45 \mathrm{e}+13$ & 0 & -500 \\
\hline 17 & $\mathrm{OH}+\mathrm{H}_{2} \mathrm{O}_{2} \rightleftharpoons \mathrm{HO}_{2}+\mathrm{H}_{2} \mathrm{O}$ & $2.00 \mathrm{e}+12$ & 0 & 427 \\
\hline 18 & $\mathrm{OH}+\mathrm{H}_{2} \mathrm{O}_{2} \rightleftharpoons \mathrm{HO}_{2}+\mathrm{H}_{2} \mathrm{O}$ & $1.70 \mathrm{e}+18$ & 0 & 29410 \\
\hline 19 & $\mathrm{OH}+\mathrm{CO} \rightleftharpoons \mathrm{H}+\mathrm{CO}_{2}$ & $4.76 \mathrm{e}+07$ & 1.23 & 70 \\
\hline 20 & $2 \mathrm{HO}_{2} \rightleftharpoons \mathrm{O}_{2}+\mathrm{H}_{2} \mathrm{O}_{2}$ & $1.30 \mathrm{e}+11$ & 0 & -1630 \\
\hline 21 & $2 \mathrm{HO}_{2} \rightleftharpoons \mathrm{O}_{2}+\mathrm{H}_{2} \mathrm{O}_{2}$ & $4.20 \mathrm{e}+14$ & 0 & 12000 \\
\hline 22 & $\mathrm{HO}_{2}+\mathrm{CO} \rightleftharpoons \mathrm{OH}+\mathrm{CO}_{2}$ & $1.50 \mathrm{e}+14$ & 0 & 23600 \\
\hline 23 & $\mathrm{OH}+\mathrm{HO}_{2} \rightleftharpoons \mathrm{O}_{2}+\mathrm{H}_{2} \mathrm{O}$ & $5.00 \mathrm{e}+15$ & 0 & 17330 \\
\hline
\end{tabular}

- 2D simulations: The results of CNN-DNS are compared to that of the detailed GRI-3.0 and the 11-species reduced mechanims of Table 2 in Figs. 5 and 6 (streamwise and spanwise distributions). Statistical means are computed after the flow is established (10 flow through times). The averaged streamwise velocity distribution along the planar fuel jet centerline decreases until $x \approx 8 \mathrm{~mm}$ (Fig. 5), location where the fuel ignites and

agation [11, 37] and a genetic algorithm [38]. The reaction rates of the reduced mechanism are optimized against the response of the detailed mechanism in non-adiabatic non-premixed canonical problems involving turbulent micro-mixing as in [36]. the mass fractions of $\mathrm{CO}$ and $\mathrm{H}_{2}$ decrease. The nonpremixed turbulent flame scalar structure is as expected, the syngas fuel composed of $\mathrm{CO}$ and $\mathrm{H}_{2}$ is mainly transformed into $\mathrm{CO}_{2}$ and $\mathrm{H}_{2} \mathrm{O}$ (Fig. 5), with a slowing down of chemistry close to the cooled wall. Because of the overall fuel-lean character of the system, the mass fraction of $\mathrm{O}_{2}$ increases along the centreline.

According to Figs. 5 and 6 , the coupled CNN-DNS reproduces very well the scalar structure of the nonpremixed planar jet flame, with a close agreement against the same DNS with the detailed GRI-3.0 mechanism. Interestingly, for the same set of species transported with the flow, the reduced mechanism tends to over-predicts the mass fraction of $\mathrm{H}$ radical while the CNN-DNS keeps a close agreement with the reference detailed mechanism (Fig. 6). The prediction of the radical $\mathrm{H}$ chemical source may actually benefit from the image-type treatment by $\mathrm{CNN}$, in which the $2 \mathrm{D}$ distribution of species is used as input. The averaged departure of wall heat flux between detailed and CNN (respectively reduced) chemistry is $3 \%$ (respectively $-1 \%$ ). With the CNN, the standard deviation of the departure represents $6.5 \%$ of the mean heat flux.

- 3D simulations: The statistics of the 3D run with CNN and the reduced mechanism are initialized from scratch and collected from 1 flow though time, with additional averaging over the 128 spanwise planes, after the initial condition has been evacuated from the computational domain and the turbulence is fully developed. Thereby the memory of the initial condition cannot affect the results. Even though it is sufficient for training, the twodimensional channel turbulence lacks vortex stretching and the three-dimensional turbulent flow cascade differs in $3 \mathrm{D}$, modifying the turbulence/chemistry interaction and the scalar averages, as seen comparing Figs. 6 and 7 . The predictions of the CNN-DNS still match those by the reduced mechanism DNS, but for $\mathrm{H}$ mass fraction, the reduced mechanism predicting again a higher level than the CNN. Based on above comparisons against GRI-3.0 in 2D, it is legitimate to assume that the scalar flame structure obtained with the CNN chemistry is actually closer to the one that would be seen in $3 \mathrm{D}$ with the detailed mechanism (out of reach with actual computing power). The averaged departure of wall heat flux between CNN and reduced chemistry is $14 \%$, with a standard deviation of $13.8 \%$ of the mean flux.

The comparison of the computational costs due to chemistry for advancing the thermochemical variables, thus excluding the flow transport part, shows that CNNchemistry is 11 times faster than the GRI-3.0 detailed mechanism, and 1.35 times faster than the 11-species reduced mechanism, the latter mainly because the Ar- 

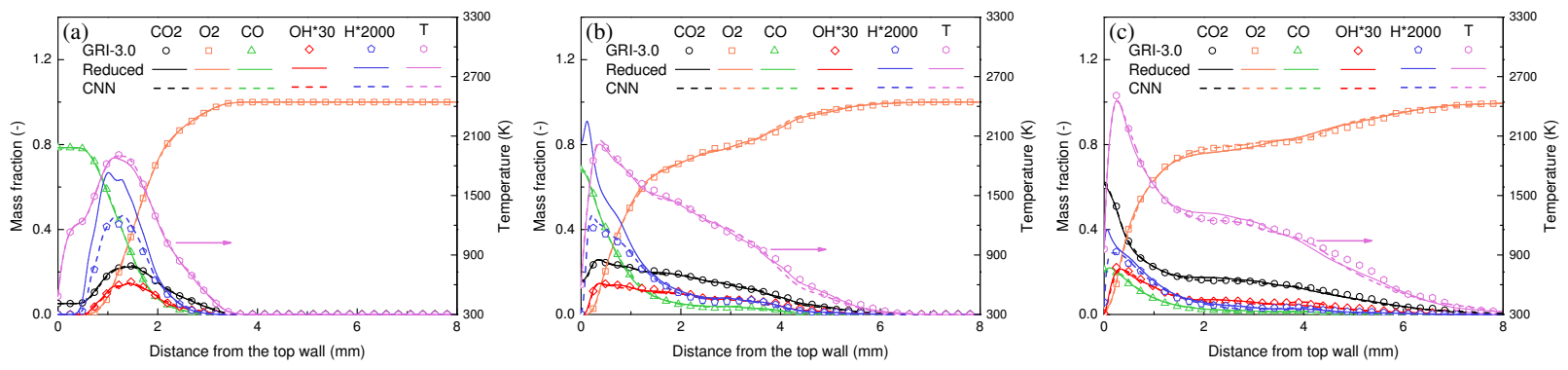

Figure 6: Spanwise averaged distributions of $\mathrm{CO}_{2}, \mathrm{O}_{2}, \mathrm{CO}, \mathrm{OH}, \mathrm{H}$ mass fractions and temperature. Symbols: GRI-3.0. Solid line: Reduced mechanism (Table 2). Dashed line: CNN chemistry. (a): $15 \mathrm{~mm}$ from the nozzle inlet. (b): $50 \mathrm{~mm}$ from the nozzle inlet. (c): $80 \mathrm{~mm}$ from the nozzle inlet. $2 \mathrm{D}$ case.
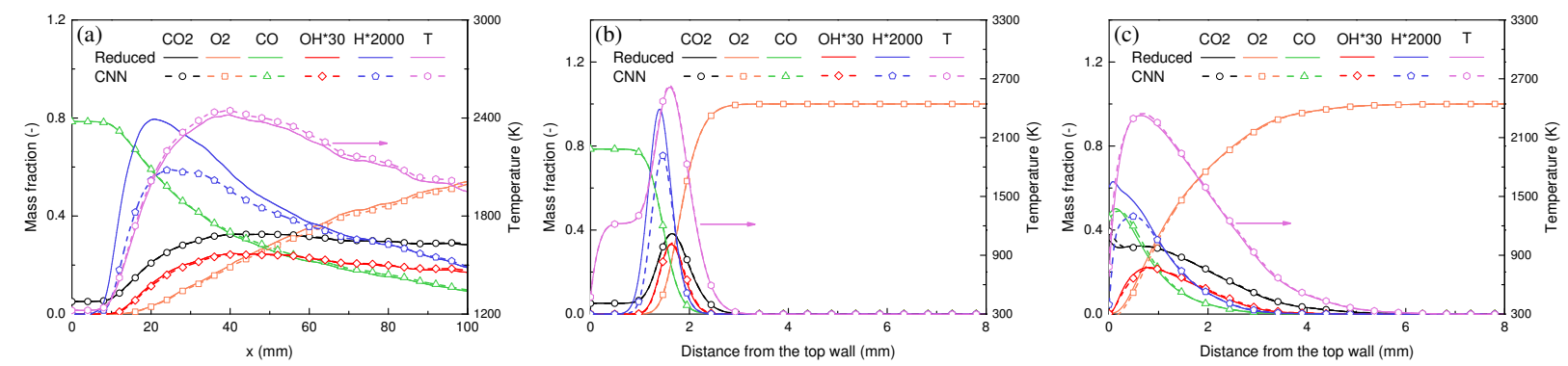

Figure 7: Averaged distributions of $\mathrm{CO}_{2}, \mathrm{O}_{2}, \mathrm{CO}, \mathrm{OH}, \mathrm{H}$ mass fractions and temperature. Solid line: Reduced mechanism (Table 2). Line with symbols: CNN chemistry. (a): BFG jet centerline (streamwise). (b): $15 \mathrm{~mm}$ from the nozzle inlet (spanwise). (c): $80 \mathrm{~mm}$ from the nozzle inlet (spanwise). 3D case.

rhenius rates calculation is avoided.

\section{Summary}

A new paradigm grounded on machine learning is discussed to introduce complex chemistry in turbulent flame simulation and implemented in three-dimensional direct numerical simulation of a syngas non-premixed flame/wall interaction. The training of a convolutional neural network (CNN) is performed on a simplified twodimensional simulation with a reference detailed chemistry. The obtained reduced chemistry is then applied to a three-dimensional turbulent flame simulation.

The comparisons against the detailed chemistry response, a reduced mechanism and the $\mathrm{CNN}$ confirm the validity of the approach, which allows for conveniently developing reduced chemistry specialized for given operating conditions from a generic detailed scheme and even save CPU time compared to using reduced mechanisms based on Arrhenius rates.

One major drawback of the presented approach lies in the need to simulate a simplified two-dimensional version of the target three-dimensional flow geometry to train the CNN. However, in future works, generic training procedures will certainly emerge, so that actually performing a flow simulation will not be mandatory to optimize the neural networks. Along these lines, the use of non-premixed stochastic micro-mixing model problems appears as a promising alternative to perform $\mathrm{CNN}$ training in a pre-processing mode. Because it has already been successfully used as a canonical problem, prior to any flow simulation, in the context of automated chemistry reduction [13].

\section{Acknowledgments}

This work is supported by ADEME (Agence de l'Environnement et de la Maîtrise de l'Energie) under the project IGAR (Injection de Gaz de Recycle) Grant $\mathrm{Nb}$ 1882C001. Computing resources were provided by CRIANN (http://www.criann.fr).

\section{References}

[1] H. J. Curran, Developing detailed chemical kinetic mechanisms for fuel combustion, Proc. Combust. Inst. 37 (2019) 57-81.

[2] X. Cheng, H. K. Ng, S. Gan, J. H. Ho, K. M. Pang, Development and validation of a generic reduced chemical kinetic mechanism for CFD spray combustion modelling of biodiesel fuels, Combust. Flame 162 (2015) 2354-2370. 
[3] N. Peters, in: Numerical simulation of combustion phenomena, Springer-Verlag, Berlin, 1985, pp. 90-109.

[4] W. P. Jones, R. P. Lindstedt, Global reaction schemes for hydrocarbon combustion, Combust. Flame 73 (1988) 233-249.

[5] D. Bradley, L. K. Kwa, A. K. C. Lau, M. Missaghi, S. B. Chin, Laminar flamelet modeling of recirculating premixed methane and propane-air combustion, Combust. Flame 71 (1988) 109122.

[6] U. Maas, S. Pope, Simplifying chemical kinetics: Intrinsic lowdimensional manifolds in composition space, Combust. Flame 88 (1992) 239-264

[7] S. H. Lam, D. A. Goussis, The CSP method for simplifying kinetics, Int. J. Chem. Kinet. 26 (1994) 461-486.

[8] J. van Oijen, F. Lammers, L. de Goey, Modeling of complex premixed burner systems by using flamelet-generated manifolds, Combust. Flame 127 (2001) 2124-2134

[9] V. Bykov, U. Maas, The extension of the ILDM concept to reaction-diffusion manifolds, Combust. Theory Model. 11 (2007) 839-862.

[10] W. P. Jones, S. Rigopoulos, S. Smith, U. Maas, Reduction of comprehensive chemistry via constraint potentials, Proc. Combust. Inst. 30 (2003) 1325-1331

[11] P. Pepiot-Desjardins, H. Pitsch, An efficient error-propagationbased reduction method for large chemical kinetic mechanisms, Combust. Flame 154 (2008) 67-81.

[12] P. Boivin, C. Jiménez, A. L. Sánchez, F. A. Williams, An explicit reduced mechanism for $\mathrm{H}_{2}$ air combustion, Proc. Combust. Inst. 33 (2011) 517-523.

[13] N. Jaouen, L. Vervisch, P. Domingo, G. Ribert, Automatic reduction and optimisation of chemistry for turbulent combustion modelling: Impact of the canonical problem, Combust. Flame 175 (2017) 60-79.

[14] P. Koniavitis, S. Rigopoulos, W. Jones, Reduction of a detailed chemical mechanism for a kerosene surrogate via RCCE-CSP, Combust. Flame 194 (2018) 85-106.

[15] Y. Chang, M. Jia, B. Niu, Z. Xu, Z. Liu, Y. Li, M. Xie, Construction of a skeletal oxidation mechanism of n-pentanol by integrating decoupling methodology, genetic algorithm, and uncertainty quantification, Combust. Flame 194 (2018) 15-27.

[16] A. Scholtissek, P. Domingo, L. Vervisch, C. Hasse, A selfcontained composition space solution method for strained and curved premixed flamelets, Combust. Flame. 207 (2019) 342355.

[17] X. Wen, Y. Luo, H. Wang, K. Luo, H. Jin, J. Fan, A three mixture fraction flamelet model for multi-stream laminar pulverized coal combustion, Proc. Combust. Inst. 37 (2019) 2901-2910.

[18] F. Christo, A. Masri, E. Nebot, S. Pope, An integrated $\mathrm{PDF} /$ neural network approach for simulating turbulent reacting systems, Symp. (Int.) Combust. 26 (1996) 43-48.

[19] L. L. Franke, A. K. Chatzopoulos, S. Rigopoulos, Tabulation of combustion chemistry via Artificial Neural Networks (ANNs): Methodology and application to LES-PDF simulation of Sydney flame L, Combust. Flame 185 (2017) 245-260.

[20] Y. LeCun, Y. Bengio, G. Hinton, Deep learning, Nature 521 (2015) 436-444.

[21] Z. M. Nikolaou, C. Chrysostomou, L. Vervisch, S. Cant, Progress Variable Variance and Filtered Rate Modelling Using Convolutional Neural Networks and Flamelet Methods, Flow Turbul. Combust. 103 (2019) 485-501.

[22] C. J. Lapeyre, A. Misdariis, N. Cazard, D. Veynante, T. Poinsot, Training convolutional neural networks to estimate turbulent sub-grid scale reaction rates, Combust. Flame 203 (2019) 255264.

[23] A. Seltz, P. Domingo, L. Vervisch, Z. M. Nikolaou, Direct mapping from LES resolved scales to filtered-flame generated mani- folds using convolutional neural networks, Combust. Flame 210 (2019) 71-82.

[24] G. P. Smith, D. M. Golden, M. Frenklach, N. W. Moriarty, B. Eiteneer, M. Goldenberg, C. T. Bowman, R. K. Hanson, S. Song, W. C. Gardiner Jr., V. V. Lissianski, Z. Qin, GRI-Mech 3.0, http://www.me.berkeley.edu/gri_mech/ 1999.

[25] J. C. R. Hunt, A. A. Wray, P. Moin, Eddies, stream, and convergence zones in turbulent flows, Cent. Turbul. Res. Rep. CTRS88 (1988) 193-208.

[26] A. Gruber, R. Sankaran, E. R. Hawkes, J. H. Chen, Turbulent flamewall interaction: a direct numerical simulation study, J. Fluid Mech. 658 (2010) 5-32.

[27] K. D. Wan, L. Vervisch, J. Xia, P. Domingo, Z. H. Wang, Y. Z. Liu, K. F. Cen, Alkali metal emissions in an early-stage pulverized-coal flame: DNS analysis of reacting layers and chemistry tabulation, Proc. Combust. Inst. 37 (2019) 27912799.

[28] K. D. Wan, J. Xia, Z. H. Wang, L. C. Wrobel, K. F. Cen, OnlineCPD-coupled large-eddy simulation of pulverized-coal pyrolysis in a hot turbulent nitrogen jet, Combust. Sci. Technol. 189 (2017) 103-131.

[29] K. D. Wan, J. Xia, Z. H. Wang, M. Pourkashanian, K. F. Cen, Large-eddy Simulation of Pilot-assisted Pulverized-coal Combustion in a Weakly Turbulent Jet, Flow Turbul. Combust. 99 (2017) 531-550

[30] K. D. Wan, J. Xia, L. Vervisch, Y. Z. Liu, Z. H. Wang, K. F. Cen, Modelling alkali metal emissions in large-eddy simulation of a preheated pulverised-coal turbulent jet flame using tabulated chemistry, Combust. Theory Model. 22 (2018) 203-236.

[31] K. D. Wan, Z. H. Wang, J. Xia, L. Vervisch, P. Domingo, Y. Lv, Y. Z. Liu, Y. He, K. F. Cen, Numerical study of $\mathrm{HCl}$ and $\mathrm{SO} 2$ impact on sodium emissions in pulverized-coal flames, Fuel 250 (2019) 315-326.

[32] C. D. Pierce, P. Moin, Progress-variable approach for largeeddy simulation of non-premixed turbulent combustion, J. Fluid Mech. 504 (2004) 73-97.

[33] O. Desjardins, G. Blanquart, G. Balarac, H. Pitsch, High order conservative finite difference scheme for variable density low Mach number turbulent flows, J. Comput. Phys. 227 (2008) 7125-7159.

[34] H. H. Aghdam, E. J. Heravi, Guide to convolutional neural networks, Springer, New York, 2017.

[35] D. P. Kingma, J. Ba, Adam: A Method for Stochastic Optimization, arXiv e-prints (2014) arXiv:1412.6980.

[36] N. Jaouen, L. Vervisch, P. Domingo, Auto-thermal reforming (ATR) of natural gas: An automated derivation of optimised reduced chemical schemes, Proc. Combust. Inst. 36 (2017) 33213330.

[37] T. Lu, C. K. Law, A directed relation graph method for mechanism reduction, Proc. Combust. Inst. 30 (2005) 1333-1341.

[38] J. H. Holland, Adaptation in Natural and Artificial Systems: An Introductory Analysis with Applications to Biology, Control and Artificial Intelligence, MIT Press, Cambridge, MA, USA, 1992. 